\title{
Traders Network before Market Crashes
}

\author{
Rossitsa Yalamova \\ Dhillon School of Business \\ University of Lethbridge \\ Canada \\ rossitsa.yalamova@uleth.ca
}

Received: May 12, 2021. Revised: November 27, 2021. Accepted: December 18, 2021. Published: January 25, 2022.

\begin{abstract}
The goal is to reveal scale-dependent topology of the network structure of stock market participants. The correlation structure of stock price series reveals the correlation network between traders. The partition decoupling method reveals the topological structure. The relation of the structural organization to dynamical complexity involves synchronization of trading that may lead to crashes. Log-periodic oscillations of index prices as precursory patterns of crashes are hypothesized to appear as a result of information cascade and herding among traders.
\end{abstract}

Index Terms-market crash, network topology, trading, synchronization.

\section{INTRODUCTION}

Complexity has always been part of our environment and many scientific fields have dealt with complex systems, which display variation without being purely random. Complex systems tend to be high dimensional and non-linear but may exhibit low-dimensional behavior. Financial markets have been shown to be similar to complex dynamical systems (Johansen et al., 2000). The different parts of complex systems are linked and affect one another.

One of the objectives in quantifying complex systems is to explain emergent structures, self-organization. Self-organized criticality is characterized by power-law distribution of events around the phase boundary. Sornette et al. (1996) argue that scale invariance and self-similarity are the dominant concepts in the processes surrounding the October $1987 \mathrm{crash}$, which could be the result of worldwide cooperative phenomena, analogous to a critical phase transition in physics. Johansen et al. (2000) identify patterns of near-critical behavior years before market crashes. The hierarchical or cascade model of traders with "crowd" or "herd" behavior illustrates the concept of criticality, where a large proportion of the actors simultaneously decide to sell their stocks (cf. Breymann et al., 2000).

Theories of asset pricing dynamics that challenge the EMH view fluctuations as arising from underlying systematic cause that leads to increased correlations in extreme events. Plerou et al (1999) analyze the cross-correlation of stock price changes in an attempt to separate noise and interaction between two companies effect on correlation and admits the difficulty of measuring the interaction strength between two companies. I argue that stock price correlations actually reflect coordination of traders activity that might be initiated by different factors related to news or noise. At the end what determines price changes are trading orders. Therefore, I propose that price changes actually measure the interaction strength among traders especially in extreme events situation. In a recent conference paper Yalamova and McKelvey (2009) propose a theoretical model of bubble build up and market crashes as an extension of the efficient market framework The model suggests that at a critical level of information complexity, rational decision making is impeded and traders' herding increases as a result of information cascade, rule based trading and imitation.

This research will apply techniques for detection of self-organization among traders in financial time series as needed preliminary work to understanding intervention timing and dynamics. The goal is to describe the complex network of market participants and propose a theoretical model of synchronization of trading activities as a result of herding behavior in extreme market conditions. The topological scales of interaction in these networks are related to the synchronization patterns. The spectral theory of the Laplacian matrix of a graph is applied and multiresolution is achieved with wavelet decomposition.

\section{SYNCHRONIZATION OF TRADING}

Lebaron (2001) suggest that crash occurs when all traders evolve towards a single trading rule. Self-organization of traders may produce criticality and significant drawdown (crash). Intervention to mitigate self-organization may prevent crash and restore equilibrium. Planning successful intervention though requires understanding of the synchronization dynamics of the market participants' actions.

Synchronization phenomena of interacting traders are hypothesized as the cause of log-periodic oscillations of prices before significant drawdowns. The dynamical process towards synchronization may exhibit different patterns related to the topology of the complex network of agents. It has been shown that highly interconnected agents synchronize more easily than those with sparse connections (Vega et al. 2004, Oh et al. 2005, etc). Furthermore those highly connected agents form local clusters involved in a network with larger groups up to the final state where the whole population is involved.

Arenas et al. (2006) propose spectral theory of the 
Laplacian matrix of a graph for the analysis of any complex networks. Different time scales of the correlation structure reveal the dynamics towards an attractor.

I propose that each group of agents trading the same stock form the nodes of the network, each node is modeled as a phase oscillator. Synchronization model of coupled phase oscillators studied by Kuramoto (2003) assumed mean-filed interaction. If the oscillators are identical, there is only one attractor of the dynamics.

In efficient market with (identically) rational agents, randomly arriving news are incorporated in asset prices quickly. The attractor is market equilibrium, the price is "right". Random networks though synchronize differently than scale free networks that we argue develop in a bubble build up regime (Yalamova and McKelvey 2009).

The focus of this project is the market condition when self-organization and bubble build up starts, a threshold of information complexity above which random 'anomalies' can not be included in the Gaussian paradigm of the efficient market. I attempt to describe the changes of the topological structure of trader's network with spectral clustering and partition decoupling methods recently applied to the equity markets. The goal is to confirm the increasing coupling and loss of heterogeneity leading to a new attractor "the critical point" and crash.

\section{SPECTRAL CluStering}

Research in machine learning and pattern recognition utilizes a variety of methods. $\mathrm{Nq}$ et al (2001) discuss the deficiencies of Expectation Maximization algorithm based methods and present a simple clustering algorithm that show good experimental results. This algorithm is later developed by Leibon et al (2008) as a core of their Hierachical Spectral Clustering Method applied to the equities market network. Spectral clustering of a graph Laplacian associated with the correlation matrix is used to analyze partition of the U.S. equities market., e.g. sector and industry or structure of the sectorial market decomposition.

By using returns on different time scales (hopefully high frequency data) correlation matrices for different return scales are calculated with wavelets. I use the largest eigenvalues of the Laplacian of the correlation matrix to analyze the clustering of traders around different industry portfolios. I argue that the notion of sector rotation in the economy, which reflects cyclical flow of capital through the various sectors of the equities market contains behavioral component of herding and imitation in a market that is in a bubble regime, i.e. self-organization of traders towards critical point.

Revealing the dynamics of the traders' complex network in periods before and during extreme events should play an important role in the development of models of the stock market dynamics and empirically test for the emergence of the collective signals out of noise in complex systems.

Understanding of the mechanisms of self-organization of the market participants leading to bubble build up and ending either in soft-landing or a crash will allow to develop better intervention strategies and regulation policies to prevent negative outcome.
Plerou et al. (1999, 2002) among others suggest that there exist three kinds of fluctuations in stock price returns: market wide, synchronized groups and random. Identification of stock groups based on the correlation matrix eigenvalues was the subject of previous research in an attempt to reveal changes in correlation and better portfolio strategy.

The goal of this project is to take more fundamental approach to understanding the mechanisms behind marketwide synchronization of trading that leads to bubble build up regimes and significant drawdowns (crashes).

The comparative analysis of stock market data during periods of extremes is motivated by the scientific evidence in physics that complex dynamical systems reveal their properties better under stress than in normal conditions. In particular, we can learn more about a system under stress by detecting the "edge of chaos," i.e. the crossover to chaotic dynamics immediately before extreme events. Moreover, extreme events observed in financial markets are much more frequent than predicted by the Gaussian paradigm that justifies the effort to look for some other dynamics than pure randomness.

\section{PARTITION Decoupling Method}

Leibon et al. (2008) apply partition decoupling method with iterative application of partition scrubbing for the decomposition that provides multiscale decomposition of the underlying data elements. The partition scrubbing uses the partitions produced with the graph Laplacian of the correlation matrix of returns:

$$
L=I-D^{-1 / 2} \otimes \exp \left(-d^{2}\right) \otimes D^{-1 / 2}
$$

Following the SI Leibon et al. (2008) as well as $\mathrm{Ng}$ et al. (2001) I developed a simple algorithm in Matlab to calculate the eigenvalues of graph's Laplacian and then compare it to the eigenvalues of a Gaussian ensemble null model. The number of significant clusters is chosen by comparison with the random matrix to determine where the random effects show up. The number of the significant clusters is equal to the number of nonzero eigenvalues of the correlation matrix that fall below the minimum of the Laplacian of the random correlation matrix. This forms the first set of clusters.

Lower levels of partition are revealed by repeating the above steps on the correlation matrix of the cluster series until partitioning failure as described in Leibon et al (2008). This method preserves important aspects of systems complexity with multipartition description that captures dominant processes and lower order structures

The multiresolution representation of the original time series is given by wavelet decomposition. This method is previously applied to financial time series for time scale decomposition.

\section{Methodology}

Network topology plays important role in dynamic behavior. Graph theoretic analysis on traders network applies some of the above mentioned methods in Yalamova (2009) while here I focus on collective synchronization phenomena that appear in stock prices before crashes. I propose a model 
of synchronization of traders' behavior leading to log-periodic patterns of index prices documented by Sornette et all. (2001), Sornette and Johansen (1998) etc.

Multiresolution is achieved with the application of wavelet transform at 6 different scales. The graph Laplacian of the correlation matrix of returns is obtained following SI Leibon et al. (2008). Recent studies show the spectral information of the Laplacian and the synchronization dynamics relationship. The master stability equation of Pecora and Carroll (1998) and eigenvalue ratios are used to determine the stability of synchronization and the heterogeneity of the topology. Arenas et al (2006) analyze the whole eigenvalue spectrum of the Laplacian matrix revealing many aspects of the topological structure, e.g. the number of disconnected components, the relative difference of time scales, the existence of hubs. Moreover, the eigenvalue of the Laplacian reveal the dynamics towards synchronization through the linearized dynamics of the Kuramoto model:

$$
\frac{d \theta_{i}}{d t}=-k \sum_{j} L_{i j} \theta_{j} \quad i=1 \ldots \ldots . . N
$$

where $\theta_{i}$ is he phase of $i$ and the solution in terms of the normal modes $\varphi_{\mathrm{i}}(\mathrm{t})$ with eigenvector matrix $\mathrm{B}$ is:

$$
\varphi_{i}(t)=\sum_{j} B_{i j} \theta_{j}=\varphi_{i}(0) \exp \left(-\lambda_{i} t\right) \quad i=1 \ldots \ldots . . N
$$

This method also allows detecting when the oscillators break through the synchronization threshold forming clusters, the R1 information complexity level in Yalamova-McKelvey (2009) model.

Link between dynamics and topology can be detected analyzing the whole spectrum of the Laplacian matrix of the network graph. Following the methodology described in Arenas et al. (2006) I rank the eigenvalues of the Laplacian in ascending order. This reveals the topological structure of disconnected components, relative differences of time scales, and the existence of hubs in the network as explained in the above reference.

\section{CONCLUSIONS}

The proposed method allows following the dynamics of self-organization in the stock market that leads to bubble build up and crash. Empirical results will allow confirming the existence of patterns in market self-organization before crash. Better understanding of the dynamics will allow planning regulation and intervention strategy to mitigate the consequent action as the causes of "crash" or soft-landing are not fully understood although the differences in the patterns of price diffusion have been documented.

This method also gives a better research and quantitative tools for the "behavioral finance" supporters. The model described in Yalamova and McKelvey (2009) attempts to reconcile the differences between efficient market and behavioral finance, creating a unified framework which accommodates both fields in different regimes. The differences in the dynamics require different research methods. To the best of my knowledge topological description of trader network has not been attempted in behavioral finance, i.e. away from equilibrium market. This method will enable quantitative analysis of connectivity and herding patterns among traders. Moreover synchronization dynamics research in finance promises better understanding of cash flow and liquidity changes to avoid crisis.

\section{REFERENCES}

[1] P. Arby, P. Flandrin, M. S. Taqqu and D. Veitch, Self-similarity in Network Traffic, Wiley, New York. 1998.

[2] A. Arenas, A. Díaz-Guilera and C. J. Pérez-Vicente, "Synchronization reveals topological scales in complex networks," Phys. Rev. Lett. vol. 96, 2006, 114102.

[3] A. Arneodo, J. F. Muzy and D. Sornette, "Direct casual cascade in the stock market," European Phys. Journal B, vol. 2, 1998, pp. 277-282

[4] W. Breymann, S. Ghashghaie and P. Talkner, "A stochastic cascade model for FX dynamics," International Journal of Theoretical and Applied Finance, v. 3, \# 3, 2000, pp.357-360.

[5] A. Johansen, D. Sornette and O. Ledoit, "Crashes as critical points," Int. J. Theo. \& Appl. Finance, v. 3, \#. 2, 2000, pp. 219-255

[6] Y. Kuramoto, Chemical Oscillations, Waves and Turbulence, Mineola NY, Dover Publications, 2003.

[7] B. LeBaron, "Evolution and time horizons in an agent-based stock market," Macroeconomic Dynamics 5, 2001, pp. 225-254.

[8] G. Leibon, S. Pauls, D. Rockmore, and R. Savell, "Topological structures in the equities market network," PNAS December 30, 2008, vol. 105 no.52 20589-20594.

[9] A. Y. Ng, M. I. Jordan and Y. Weiss, “On spectral clustering: analysis and an algorithm," in Advances in Neural Information Processing Systems 14, 2001, MIT Press, Cambridge, MA

[10] E. Oh, K. Rho, H. Hong and B. Kahng, "Modular synchronization in complex networks," Phys. Rev. E 72, 2005, 047101]

[11] L. M. Pecora and T. L. Carroll, "Master stability function for synchronized coupled systems," Phys. Rev. Lett. Vol. 80, \#10, 1998, pp. 2109-2112.

[12] V. Plerou, P. Gopikrishnan, B. Rosenow, A. L. N. Amaral, and H. E.Stanley, "Universal and nonuniversal properties of cross correlations in financial time series," Phys. Rev. Lett. Vol. 83, \#7, 1999, pp. 1471-1474.

[13] V. Plerou, P. Gopikrishnan, B. Rosenow, A. L. N. Amaral, T. Guhr and H. E.Stanley, "Random matrix approach to cross correlations in financial data," Phys. Rev. E, 65, 2002, 066126.

[14] D. Sornette, A. Johansen. and J.-P. Bouchaud, "Stock market crashes, precursors and replicas," J. Phys. I France, v. 6, January 1996, pp. $167-175$.

[15] D. Sornette and A. Johansen, "A hierarchical model of financial crashes," Physica A, vol. 261(3-4), 1998, pp: 581-598

[16] Y. M. Vega, M. Vázquez-Prada and A. F. Pacheco, "Fitness for synchronization of network motifs," Physica A, vol. 343, 2004, pp. 279-287.

[17] R. Yalamova, "Non-linear dynamic research methods in finance curriculum" The 2009 College Teaching \& Learning Conference, Prague.

[18] R. Yalamova and B. McKelvey, "Explaining What Leads Up to Stock Market Crashes: A Phase Transition Model and Scalability Dynamics,” 2nd International Financial Research Forum Paris, 2009.

\section{Creative Commons Attribution License 4.0 (Attribution 4.0 International, CC BY 4.0)}

This article is published under the terms of the Creative Commons Attribution License 4.0 https://creativecommons.org/licenses/by/4.0/deed.en US 